\title{
Effectiveness of exercise programs to reduce falls in older people with dementia living in the community: a systematic review and meta-analysis
}

This article was published in the following Dove Press journal:

Clinical Interventions in Aging

9 February 2015

Number of times this article has been viewed

\author{
Elissa Burton ${ }^{1,2}$ \\ Vinicius Cavalheri' \\ Richard Adams ${ }^{3}$ \\ Colleen Oakley Browne 4 \\ Petra Bovery-Spencer ${ }^{4}$ \\ Audra M Fenton ${ }^{3}$ \\ Bruce W Campbell ${ }^{5}$ \\ Keith D Hill ${ }^{1,6}$
}

'School of Physiotherapy and Exercise Science, Curtin University, Perth, WA, Australia; ${ }^{2}$ Research Department, Silver Chain, Perth, WA, Australia; ${ }^{3}$ Community Services, West Gippsland Healthcare Group, Warragul, VIC, Australia; ${ }^{4}$ Falls Prevention for People Living with Dementia Project, Central West Gippsland Primary Care Partnership, Moe, VIC, Australia; ${ }^{5}$ Allied Health, Latrobe Regional Hospital, Traralgon, VIC, Australia; ${ }^{6}$ Preventive and Public Health Division, National Ageing Research Institute, Melbourne, VIC, Australia

Correspondence: Elissa Burton Curtin University, School of Physiotherapy and Exercise Science, GPO Box U 1987. Perth,WA 6845, Australia

Tel +6I 89266368 I

Fax +6I 892663699

Email e.burton@curtin.edu.au
Objective: The objective of this systematic review and meta-analysis is to evaluate the effectiveness of exercise programs to reduce falls in older people with dementia who are living in the community.

Method: Peer-reviewed articles (randomized controlled trials [RCTs] and quasi-experimental trials) published in English between January 2000 and February 2014, retrieved from six electronic databases - Medline (ProQuest), CINAHL, PubMed, PsycInfo, EMBASE and Scopus according to predefined inclusion criteria were included. Where possible, results were pooled and meta-analysis was conducted.

Results: Four articles (three RCT and one single-group pre- and post-test pilot study) were included. The study quality of the three RCTs was high; however, measurement outcomes, interventions, and follow-up time periods differed across studies. On completion of the intervention period, the mean number of falls was lower in the exercise group compared to the control group (mean difference $[\mathrm{MD}][95 \%$ confidence interval $\{\mathrm{CI}\}]=-1.06[-1.67$ to -0.46$]$ falls). Importantly, the exercise intervention reduced the risk of being a faller by $32 \%$ (risk ratio [95\% $\mathrm{CI}]=0.68$ [0.55-0.85]). Only two other outcomes were reported in two or more of the studies (step test and physiological profile assessment). No between-group differences were observed in the results of the step test (number of steps) ( $\mathrm{MD}[95 \% \mathrm{CI}]=0.51[-1.77$ to 2.78$]$ ) or the physiological profile assessment (MD $[95 \% \mathrm{CI}]=-0.10[-0.62$ to 0.42$])$.

Conclusion: Findings from this review suggest that an exercise program may potentially assist in preventing falls of older people with dementia living in the community. However, further research is needed with studies using larger sample sizes, standardized measurement outcomes, and longer follow-up periods, to inform evidence-based recommendations.

Keywords: cognitive impairment, older people, physical activity, fallers, community dwelling

\section{Introduction}

Dementia is a major health issue predominantly affecting older people. It is estimated that over 44 million people worldwide are living with dementia, and by 2050 there may be as many as 135.5 million people diagnosed with dementia. ${ }^{1}$ The world population is aging and as such it is expected that the increase in the number of older people will correspond with an increase in the number of older people living with dementia (PLWD). Dementia is a syndrome that impairs brain function and cognition. As the severity of dementia increases over time, the person with dementia often has increased difficulties with many important functions, including gait impairments, problems with postural control, reduced participation in activities such as shopping and driving, 
and an increase in disability leading to difficulties in eating, bathing, and dressing. ${ }^{2,3}$ The impairments in cognition, gait, and postural control also increase the risk of falls in people with dementia. Approximately $30 \%$ of adults aged 65 years and over living in the community experience one or more fall each year, ${ }^{4}$ but up to $50 \%-80 \%$ of PLWD fall in a 12-month period. ${ }^{5,6}$ There are many identified risk factors for falls, including intrinsic factors such as postural instability (gait and balance impairments), medications, neurocardiovascular complications, and vision impairment, as well as extrinsic factors such as the environment (curbs, rugs, or poor lighting). ${ }^{7}$ Falls can often lead to a fear of falling or loss of confidence, which may result in a decline in activity and ultimately a decrease in strength, balance, and mobility, leading to decreased functional ability and a loss of independence. ${ }^{8,9}$ Falls are also often a trigger for emergency department or hospital admission for older people with dementia ${ }^{10}$ and/or admission to residential care. ${ }^{10,11}$

Balance and mobility impairments in older people have been shown to be a strong independent risk factor for falling, ${ }^{12}$ and have been shown to decline at a significantly faster rate in PLWD than age-matched older people without cognitive impairment. ${ }^{13}$ To combat postural instability and decreases in function, a large number of studies have been conducted, investigating the effectiveness of exercise or physical activity programs to prevent falls for older people with a history of falling. ${ }^{4,14}$ Reviews of these studies have shown that strength- and balance-focused exercise programs have been successful in decreasing the rate of falls for older people living in the community with no cognitive impairment, using both group- and home-based environments for exercise. Based on these results, exercise or physical activity programs are viewed as an important part of falls prevention programs. ${ }^{3,4,15}$ However, direct translation of falls prevention programs that have been shown to be effective in reducing falls in samples with no cognitive impairment (eg, Close et $\mathrm{al}^{16}$ multifactorial intervention) may not be effective when implemented with people with cognitive impairment. ${ }^{17}$

Despite the higher risk of falls and greater rate of decline of balance and gait in PLWD in the community, there has been only a small but growing amount of research investigating the effect of exercise on improving physical performance and reducing falls in people with dementia. There have been nine systematic reviews ${ }^{14,18-25}$ and four general reviews ${ }^{7,17,26,27}$ investigating the effects of dementia/Alzheimer's disease on falls, and eight systematic reviews exploring the effects of exercise (types of exercise) on people with cognitive impairment..$^{3,28-34} \mathrm{Six}$ of the 21 reviews reported earlier included studies using PLWD in a residential care or hospital setting, four had a majority of participants living in the community, nine had a mix of older people living in both community and residential care, and three were unclear in describing the setting. Of the four reviews where older people with dementia living in the community setting were the majority sample population, one focused on medicine and falls, ${ }^{21}$ another recruited participants living in the community from health care settings (emergency department, dementia specific service, etc), ${ }^{25}$ the third looked at falls risk with no specific emphasis on exercise, ${ }^{19}$ and the fourth investigated the relationship between executive function, falls, and gait abnormalities. ${ }^{32}$ There is a dearth of research that specifically explored falls prevention exercise interventions for people with dementia (cognitive impairment) living in the community.

One systematic review by Hauer et $\mathrm{al}^{35}$ did investigate the effectiveness of physical training on motor performance and fall prevention in cognitively impaired older persons (search strategy was between 1966 and 2004). Again, of the eleven randomized controlled trials (RCTs) included in this review, nine were from a residential/long-term care/hospital setting and one was of people living in the community, and in the other the setting was unknown. Physical training was not defined and appeared to represent strength and balance programs designed by physiotherapists to be conducted in either an individual or group setting. ${ }^{35}$ Physical activity programs such as Tai Chi, which have been shown to be effective in reducing falls, were not included.

In summary, to date the reviews published in this area are limited when exploring those that have specifically focused on older PLWD in the community with exercise and/or physical activity as the intervention, and with falls as the outcome of interest. This systematic review seeks to address this gap. The purpose of this review is to evaluate the available evidence on the effectiveness of exercise or physical activity programs to reduce falls in older people with dementia who are living in the community.

\section{Method}

\section{Eligibility criteria}

The review is limited to studies meeting the following eligibility criteria:

- aged 60 years and over (at least $50 \%$ of the sample size);

- living in the community;

- PLWD or cognitively impaired. Dementia had to have been identified by diagnosis by a doctor/specialist, or a validated test, such as the Mini Mental State Examination 
(MMSE), the Clinical Dementia Rating Scale, or the National Institute of Neurological and Communicative Disorders and Stroke, Alzheimer's Disease and Related Disorders Association (NINCDS-ADRDA) Alzheimer's criteria;

- an exercise or physical activity program (intervention) targeting a reduction in falls (and/or) risk of falls;

- outcome measures, which include number of falls, rate of falls, or number of fallers, or time to first fall. Other outcomes of interest were fear of having a fall, functional, physical performance (eg, balance or mobility), or cognitive benefits, or adherence to exercise/physical activity intervention;

- study design: RCTs and quasi-experimental trials.

\section{Information sources}

Studies were identified by searching six databases (Medline [ProQuest], CINAHL, PubMed, PsycInfo, EMBASE, and Scopus, from January 2000 to February 2014). The search strategy commenced from 2000, given a detailed review by Hauer et $\mathrm{a}^{35}$ which searched across residential care, hospital, and community settings, and did not identify any relevant papers prior to 2003 in the community setting. In addition, reference lists of the identified papers were scanned. Only papers in English were included, no unpublished data, books, conference proceedings, theses, or poster abstracts were included.

\section{Search strategy}

The search was conducted using a mix of keywords to be identified in the abstract and/or title of the paper or MESH terms. The search strategy undertaken in Medline is presented in Table 1. Each search was limited to English language and the time period of January 1, 2000, to February 2014. Language and syntax were adapted to individual databases: for example, PubMed allowed title/abstract searches but not all databases allowed this, so in these cases only the abstract was searched.

\section{Study selection}

The study selection was conducted in three stages: stage one involved the first author (EB) initially screening the titles and scanning abstracts against the inclusion criteria to identify relevant articles. This was followed (stage two) by a full screening of the abstracts by EB. Stage three included screening of the full articles by two of the authors (EB and $\mathrm{KH})$ to identify whether they met the eligibility criteria. Any disagreements regarding potential inclusion were resolved by
Table I Search strategy (according to Medline terminology)

\begin{tabular}{ll}
\hline I & cognitive* impair* ti,ab. \\
2 & cognition disorders/[MESH] \\
3 & dementia/[MESH] \\
4 & I or 2 or 3 \\
5 & physical activity ti,ab. \\
6 & physical active* ti,ab. \\
7 & physical exerc* ti,ab. \\
8 & exercise* ti,ab. \\
9 & 5 or 6 or 7 or 8 \\
10 & community* ti,ab. \\
11 & home ti,ab. \\
12 & I0 or II \\
13 & fall* ti,ab. \\
14 & accidental fall/[MESH] \\
15 & fall* prevent* ti,ab. \\
16 & I3 or 14 or 15 \\
17 & 4 and 9 and 12 and 16 \\
\hline
\end{tabular}

discussion between EB and $\mathrm{KH}$ to achieve consensus, after referring to the eligibility criteria and protocol.

The PRISMA checklist was used to ensure that the results were reported systematically. ${ }^{36}$

\section{Data collection process}

Each study included in this review was evaluated, and the following data were extracted: study design, purpose, intervention, sample size, sex proportion, age of participants, dropout rate of participants, MMSE or rating of dementia score, number of fallers, time to first fall, fear of having a fall, measures of balance, mobility, and function, intervention effect, and length of follow-up.

\section{Study quality}

Methodological quality was assessed using the Cochrane Collaboration's risk of bias tool by two independent researchers (EB and $\mathrm{KH}) \cdot{ }^{37} \mathrm{~A}$ third independent researcher completed the risk of bias tool for one of the included studies because of a conflict of interest for $\mathrm{KH} .{ }^{38}$ The categories assessed were sequence generation, allocation concealment, blinding of participants and staff, blinding of outcome assessment, incomplete outcome data, selective outcome reporting, and other sources of bias. ${ }^{37}$ Risk of bias was assessed to be "low risk", "unclear risk", or "high risk" of bias. ${ }^{37}$

\section{Data analysis}

The studies are described according to their characteristics, interventions utilized, outcome measures, adherence to exercise interventions, study quality, and effectiveness of the intervention programs. 
Three continuous outcomes (mean falls, step test, and physiological profile assessment [PPA]) and one dichotomous outcome (faller status [ie, faller versus non-faller]) were included in the quantitative analyses. The mean difference (MD) and 95\% confidence intervals (CI) were calculated for continuous outcomes, whereas risk ratio (RR) and 95\% CI were calculated for dichotomous outcomes. The Review Manager (RevMan) version 5.2 was used to conduct the analyses and generate the forest plots, and a fixed-effect model was applied. Heterogeneity was assessed by the $I^{2}$ statistic and by visual inspection of the forest plots. For continuous outcomes, the results of homogeneous studies were subjected to meta-analysis using the inverse variance DerSimonian and Laird method..$^{39}$ For faller status (ie, dichotomous outcome), the results of homogeneous studies were meta-analyzed using the Mantel-Haenszel's fixed effects model. ${ }^{40}$ Two-sided value of $P<0.05$ was the statistically significant level set for all analyses.

In instances where data provided in the published papers were insufficient for the meta-analysis, the corresponding authors for the RCT papers were contacted and asked for the total number of falls pre and post intervention, the mean number of falls per group at post intervention, the standard deviation, the number of fallers per group (post intervention), and the number of participants for both groups at pre and post data collection.

\section{Results}

\section{Study selection}

The search strategy yielded 2,279 articles from six databases. Duplicate articles within each database were removed, leaving 446 articles. The 446 articles were then combined into an excel spreadsheet, with duplicates again removed, resulting in 286 remaining articles. Articles were then screened on the basis of title, with 179 articles excluded (reasons for exclusion are reported in Figure 1). The 107 articles were then checked, and 79 articles were excluded based on the abstracts. The full manuscripts of the 28 remaining articles were then examined in detail, and 24 were found not to meet the inclusion criteria. A total of four articles were left for inclusion in the review. Three of these were RCTs and were included in the meta-analyses.

\section{Study characteristics}

Three of the four articles included in the review were RCTs ${ }^{38,41,42}$ The fourth was a single-group pre- and post-test pilot study. ${ }^{43}$ As presented in Table 2, sample sizes across the studies ranged from $22^{42}$ to 210 participants. ${ }^{41}$ Three-hundred and thirty-six participants completed pre-testing in the four studies, but only 243 completed post-testing (72.3\%). The largest dropout rate was found for Mackintosh and Sheppard's ${ }^{43}$ study, with half of the participants not continuing across the 6-month study period. In contrast, Wesson et $\mathrm{al}^{42}$ only had one participant from the intervention group drop out prior to completing the 12 -week intervention (95.2\% completed the follow-up assessment).

All studies included MMSE scores of participants, with an average score (and standard deviation) of 18.9 (5.5) across all studies. There was little difference in MMSE scores between the intervention groups (20.4 [5.1]) and controls (20.6 [5.0]) for the three RCTs. ${ }^{38,41,42}$ All studies reported the mean age and standard deviation; the average age of the participants was $79.8(5.8)$ years.

\section{Interventions}

Interventions ranged between 3 months and 12 months, and participants randomized to the intervention group were recommended to complete the exercises once a week, ${ }^{43}$ twice a week, ${ }^{41}$ three times a week, ${ }^{42}$ or five times a week. ${ }^{38}$ The exercise interventions took place in a group (or individual assistance where required) at a facility ${ }^{41,43}$ or at home. ${ }^{38,41,42}$ Pitkälä et al's ${ }^{41}$ study comprised three groups: group-based exercise, home-based exercise, and usual care (control). Two studies provided multifactorial programs, which included an exercise component. ${ }^{42,43}$ Other interventions included in the two studies were foot health, medication management, vision assessments, walking aids and footwear issues, ${ }^{43}$ and home hazard reduction. ${ }^{42}$ The exercise programs predominantly concentrated on strength, balance, and mobility, and these programs were established and supervised by physiotherapists, occupational therapists, or physiotherapy students who were trained and supervised by physiotherapists. In two of the studies, ${ }^{38,42}$ carers were actively involved in monitoring and encouraging participation between therapist visits for the assigned exercise program.

Participants in Mackintosh and Sheppard's ${ }^{43}$ study undertook lower limb strength exercises (hip abductor, knee extensor, and ankle dorsiflexion, bilaterally) using velcro ankle weights; balance exercises while standing, and walking was based on time or distance. The falls risk assessment assisted the physiotherapist to develop individual falls and injury prevention plans for each participant. Pitkälä et al ${ }^{41}$ had two exercise groups (home-based and group-based) with different programs. The home exercise group was given individually tailored exercise programs provided by a physiotherapist with specialist dementia training and the exercises addressed the 


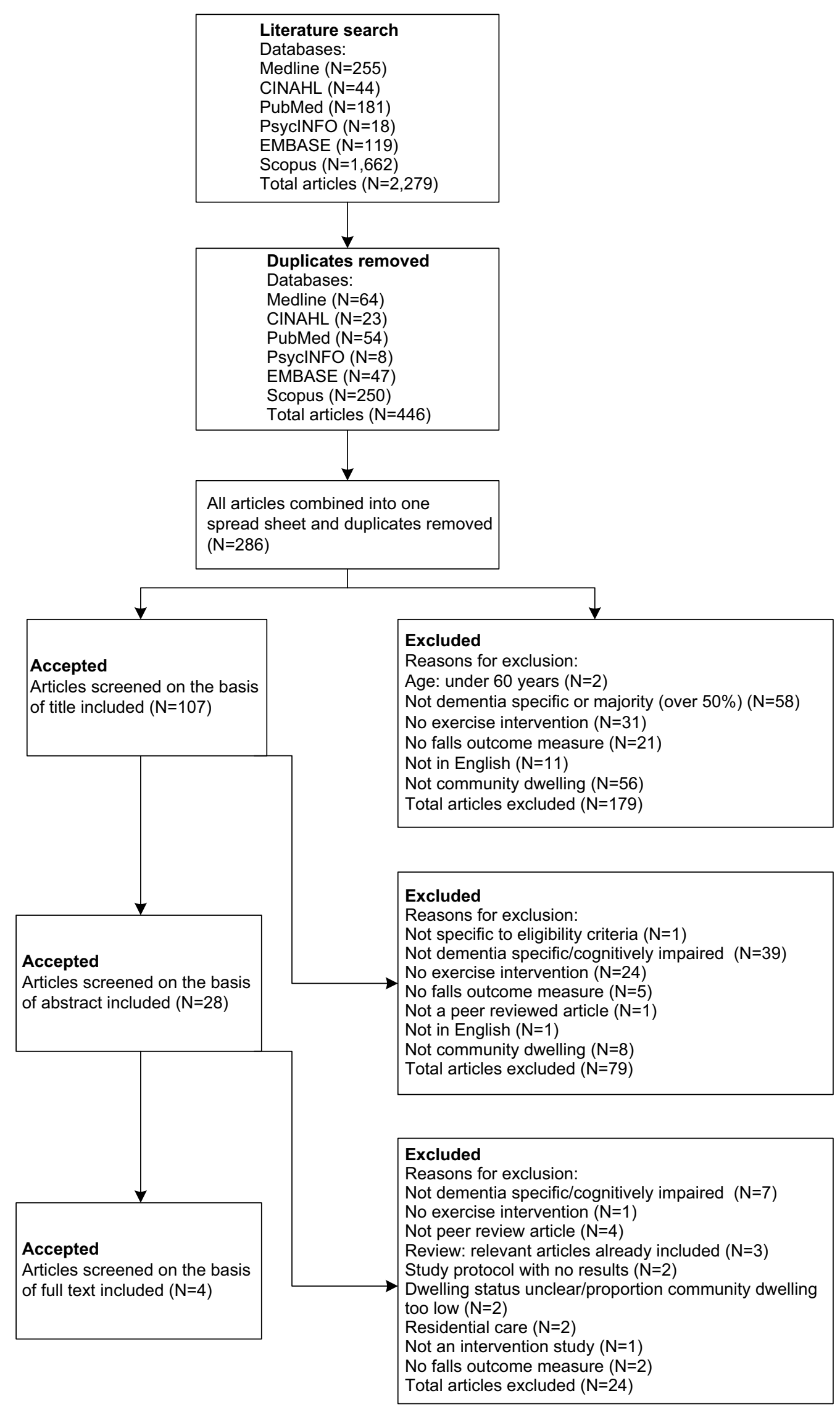

Figure I Study selection flowchart. 


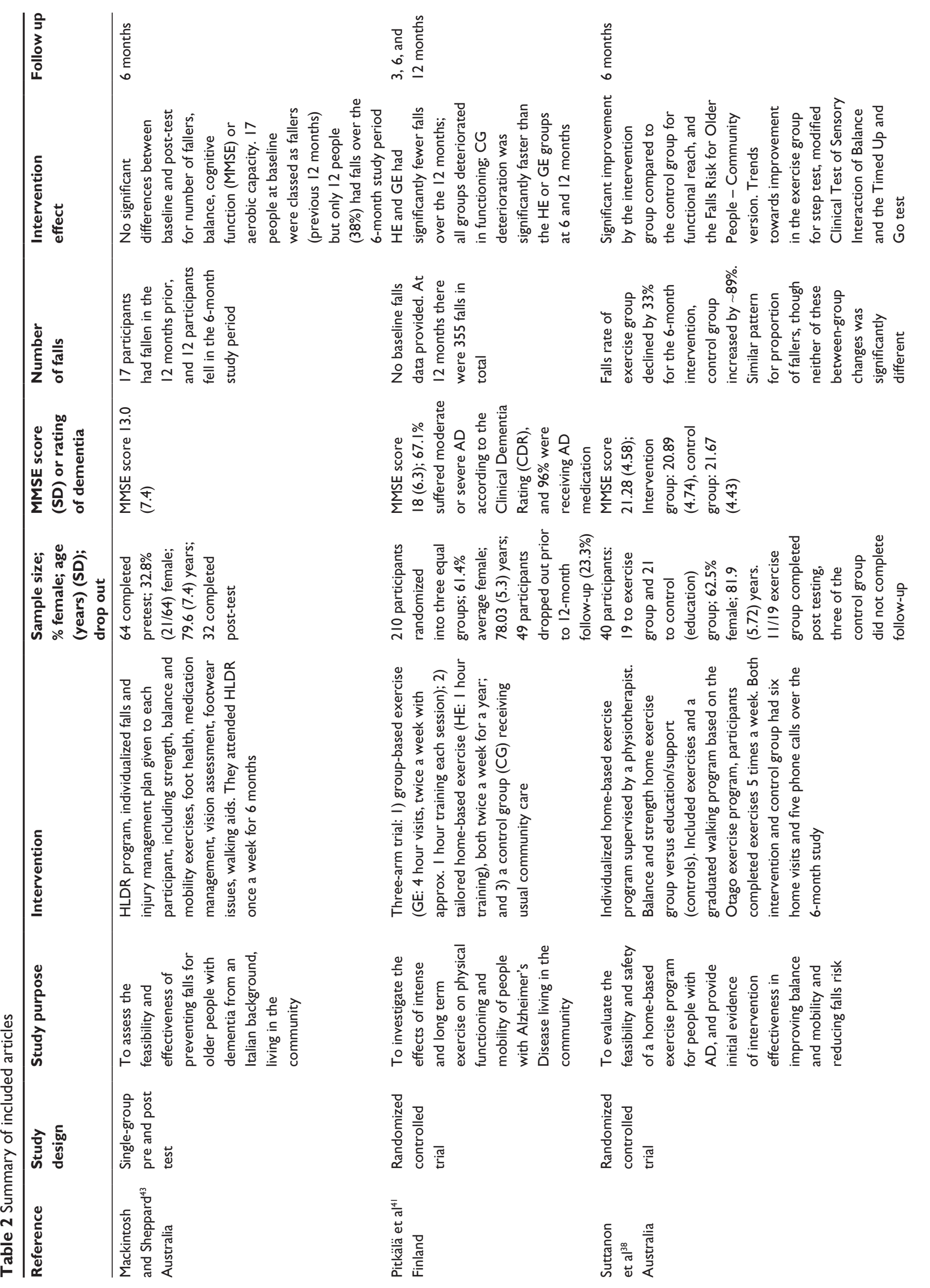




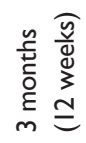
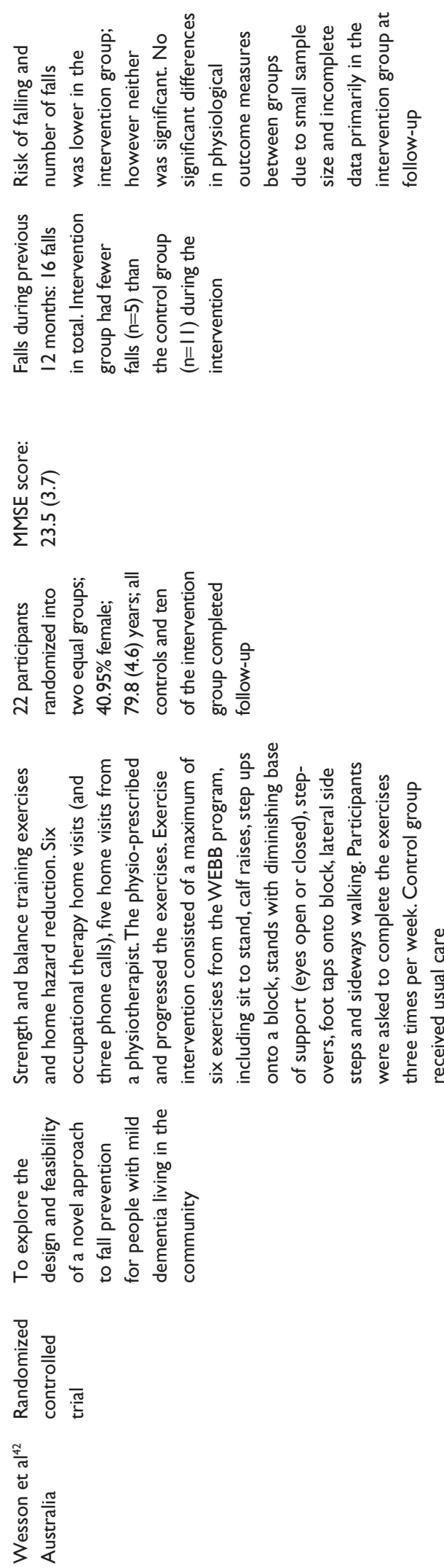

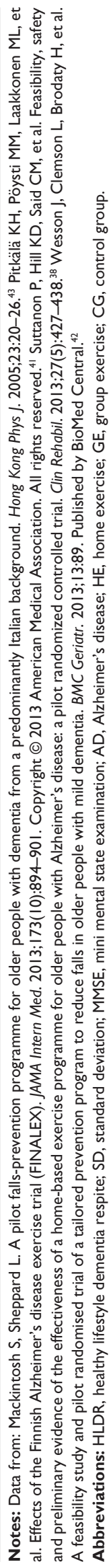

participant's needs and problems regarding daily functioning and mobility. ${ }^{41}$ Exercises included climbing stairs, balance and transfer training, walking, dual tasking, and outdoor activities. Equipment included exercise bikes, ankle/hand weights, balls, canes, and balance pillows. The group exercisers undertook predetermined exercises consisting of endurance (exercise bikes), balance (walking in a line, training while bouncing a ball, climbing a ladder), and strength training (specialized weights in the gym). All exercises increased in intensity and dosage over time and were recorded. The group ratio was ten participants to two physiotherapists.

Suttanon et $\mathrm{al}^{38}$ based their home exercise regimen on the Otago exercise program, which has been shown to reduce falls for older people living in the community. ${ }^{44}$ The exercise program included strengthening exercises, standing balance exercises, and a walking program, and meets the physical activity guidelines (30 minutes at least five times a week) recommended by the World Health Organization and the Australian Government. ${ }^{45,46}$ Ankle weights were used to progress the strengthening exercises over time, and each participant also received a booklet with illustrations and instructions of the exercises. A physiotherapist conducted six home visits over the 6-month intervention (four in the first two months) ${ }^{47}$ to prescribe individualized exercises based on assessment findings and to ensure that the carer and the person with dementia understood the exercises and safety issues, and subsequent visits were used to monitor, modify exercises if required, and motivate the participant. The carer supervised the exercise program on a day-to-day basis.

Wesson et $\mathrm{al}^{42}$ utilized strength and balance exercises from the Weight-Bearing Exercise for Better Balance (WEBB) program. ${ }^{48} \mathrm{Up}$ to six exercises were individually prescribed by a physiotherapist for each participant based on their physical performance assessment. Depending on the participant's cognitive level, they were fully supervised, or given a written booklet and visual cues around the home with supervision from a carer, or completed the exercises unsupervised. ${ }^{42}$ Exercises included sit to stand, calf raises, step-ups onto a block, stances with reduced base of support (eyes open and closed), step-overs, foot taps onto a block, lateral sidesteps, and sideways walking. ${ }^{42}$ The number and intensity of the exercises were increased as the participant progressed.

\section{Outcome measures}

There were a wide range of measurements used across the four studies. Falls were reported as number of falls, number of people falling, and rate of falls. Falls risk was assessed 
using the Falls Risk for Older People - Community version (FROP-Com) questionnaire, and the PPA. Table 3 presents the falls and physical measurement tools utilized by the four studies, and also shows whether a significant difference between the groups was found in any study (see asterisk). Three of the four studies reported the number of falls, ${ }^{41-43}$ and two the rate of falling. ${ }^{38,41}$ Apart from these and the PPA $^{38,42}$ and Hill Step Test, ${ }^{38,42}$ no other (physical or falls efficacy) measurements (or tools) were used in more than one study.

\section{Dropouts and adherence to exercise interventions}

Dropout rates varied between $4.5 \%(n=1)^{42}$ and $50 \%(n=32){ }^{43}$ Each study reported reasons for clients withdrawing, and these included Mackintosh and Sheppard: ${ }^{43}$ admitted to residential care $(n=11)$, died $(n=7)$, did not complete program $(n=7)$, variety of reasons $(n=7)$; Pitkälä et al: ${ }^{34} \operatorname{died}(n=17)$, admitted to residential care $(n=18)$, impaired health $(n=6)$, spouse/caregiver died $(n=1)$, declined to continue $(n=7)$; Suttanon et al: ${ }^{38}$ refused to continue $(\mathrm{n}=5)$, admitted into residential care $(n=4)$, hospitalized $(n=1)$, died $(n=1)$; and Wesson et al: ${ }^{42}$ hospitalized $(\mathrm{n}=1)$.

Adherence to the exercise interventions varied between not being reported ${ }^{43}$ and the three RCTs providing the percentage of participant involvement in the recommended dosage across the study periods. Over $80 \%(n=9)$ of the eleven participants who completed the 6-month home exercise intervention by Suttanon et $\mathrm{al}^{36}$ completed the home-based exercise program 5 days a week for 6 months. Two participants in this study were considered to have low adherence (21.6\% and $65.2 \%$ ), which was due to an unwillingness to participate and the presence of another health condition preventing involvement. ${ }^{38}$

Pitkälä et a ${ }^{41}$ compared group and home-based exercise programs to a control group and measured adherence as participants completing at least $50 \%$ of the sessions. Almost $93 \%$ of home exercisers completed a minimum of half of the sessions compared to $78.6 \%$ of the group exercisers. The median number of sessions attended for each group was as follows: home exercise: 81 (range 7-89), and group exercise 75 (range 7-89). ${ }^{41}$ The exercise group in Wesson et al' $\mathrm{s}^{42}$ study participated an average of 2.8 times per week (range $0-7$ ) over 3 months, with almost three-quarters $(n=8)$ of the participants completing an average of 3.3 exercises per session (range $2-5$ exercises). Reasons for not participating were as follows: had not exercised in a long time prior to the study, could not exercise without supervision, short

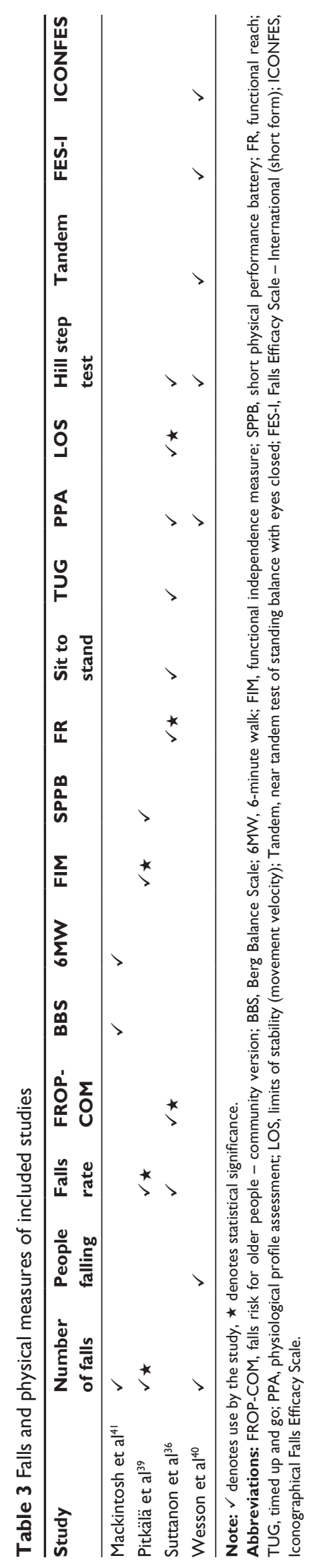


Table 4 Assessment of risk of bias for included studies

\begin{tabular}{|c|c|c|c|c|c|c|}
\hline \multirow[t]{2}{*}{ Study } & \multicolumn{2}{|c|}{ Selection bias } & \multirow{2}{*}{$\begin{array}{l}\text { Performance bias } \\
\text { Blinding of participants } \\
\text { and personnel }\end{array}$} & \multirow{2}{*}{$\begin{array}{l}\text { Attrition bias } \\
\text { Incomplete } \\
\text { outcome data }\end{array}$} & \multirow{2}{*}{$\begin{array}{l}\text { Reporting bias } \\
\text { Selective outcome } \\
\text { reporting }\end{array}$} & \multirow{2}{*}{$\begin{array}{l}\text { Other bias } \\
\text { Free of } \\
\text { other bias }\end{array}$} \\
\hline & $\begin{array}{l}\text { Sequence } \\
\text { generation }\end{array}$ & $\begin{array}{l}\text { Allocation } \\
\text { concealment }\end{array}$ & & & & \\
\hline Mackintosh and Sheppard ${ }^{41}$ & $\bullet$ & $\bullet$ & $\bullet$ & $\bullet$ & $\circ$ & $\times$ \\
\hline Pitkälä et al ${ }^{39}$ & $\circ$ & $\circ$ & $\circ$ & $\circ$ & $\circ$ & $\circ$ \\
\hline Suttanon et $\mathrm{al}^{36}$ & $\circ$ & $\circ$ & $\circ$ & $\circ$ & $\circ$ & $\circ$ \\
\hline Wesson et $\mathrm{al}^{40}$ & $\circ$ & ० & ○ & $\bullet$ & $\circ$ & $\circ$ \\
\hline
\end{tabular}

Note: Bias was scored as low risk $(\circ)$, unclear $(\times)$, or high risk $(\bullet)$.

periods of non-adherence during holidays, and brief periods of illness. ${ }^{42}$

\section{Quality of studies}

The study by Mackintosh and Sheppard ${ }^{43}$ had a number of methodological weaknesses when using the risk bias tool due to the study being a single-group pre-test, post-test trial rather than an RCT methodology. The assessed potential bias in each study is reported in Table 4. Pitkälä et al ${ }^{41}$ and Suttanon et $\mathrm{al}^{38}$ were assessed as having low risk of bias across all of the domains. Wesson et $\mathrm{al}^{42}$ did differ slightly, as it was deemed that attrition bias may have occurred, because, although the authors acknowledged missing data, it may have affected the direction of the results. The authors' reasoning for not adjusting for this in the data analysis was the "exploratory nature of the pilot study", ${ }^{42}$ and therefore bias may have occurred. All three RCTs used intention-to-treat analysis. Overall, the three RCTs were regarded as high-quality studies. ${ }^{38,41,42}$

\section{Effectiveness of intervention programs}

Even though all of the groups deteriorated in function over the year-long study in Finland, Pitkälä et $\mathrm{al}^{41}$ reported deterioration being significantly slower in the exercise groups (group-based exercise, home-based exercise) compared to the control group, for the functional independence measure (FIM; total and motor scores) at 6 months and 12 months, and that the home- and group-exercise groups had significantly fewer falls than the control group over 12 months. Suttanon et al's ${ }^{38}$ was the only other study to show a significant difference between the groups, and these were for the FROPCom $(P=0.008)$, Functional Reach $(P=0.002)$, and Limits of Stability balance measure (movement velocity, $P=0.016$ ).

Mackintosh and Sheppard ${ }^{43}$ reported a reduction in the number of people who fell (17 to 2) between pre and post testing, and Wesson et $\mathrm{a}^{42}$ reported fewer falls in the exercise group ( $\mathrm{n}=5)$ compared to the control group $(\mathrm{n}=11)$, with only two and four people having fallen from the intervention and control groups, respectively.

None of the four studies published the mean scores for the number of falls participants experienced during the intervention period. On emailing the corresponding authors from the three included RCTs for further falls data (mean pre and post number of falls, standard deviation and sample size per group), all of them responded. ${ }^{38,41,42}$ However, because of a significant difference between groups at baseline for proportion of falls, the study by Suttanon et a ${ }^{38}$ was not included in the meta-analyses.

Figure 2 reports the forest plot for the mean number of falls for the studies by Pitkälä et $\mathrm{al}^{41}$ and Wesson et $\mathrm{al}^{42}$

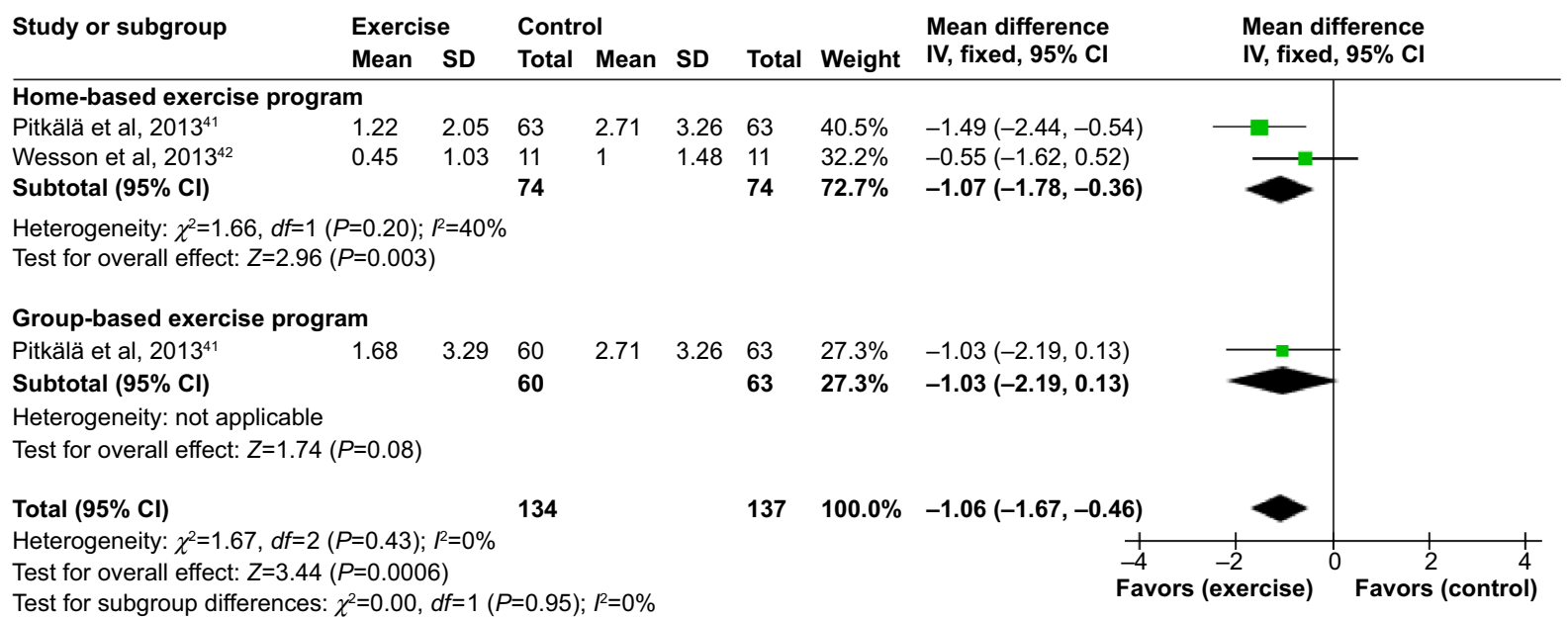

Figure 2 Forest plot of comparison: exercise versus usual care for mean number of falls. 


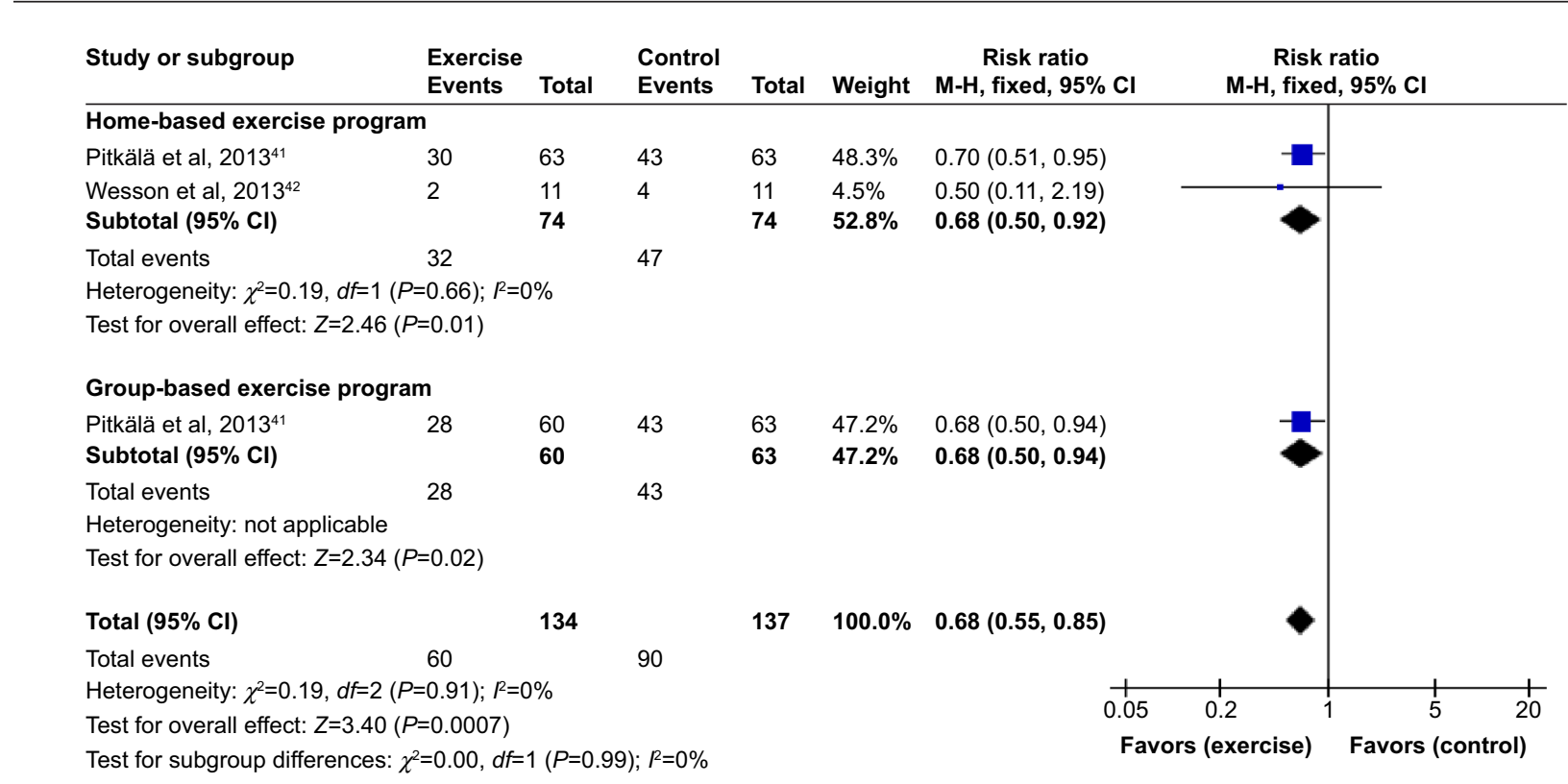

Figure 3 Forest plot of comparison: exercise versus usual care for fallers versus non-fallers. Abbreviations: $\mathrm{Cl}$, confidence interval; $\mathrm{M}-\mathrm{H}$, Mantel-Haenszel.

No overall heterogeneity was found between the two studies $\left(I^{2}=0 \%\right)$. On completion of the intervention period, mean falls were statistically lower in the exercise group compared to the control group (MD [95\% CI] $=-1.06[-1.67$ to -0.46$]$ falls). Subgroup analysis was conducted, showing that this benefit was slightly greater for home-based exercise than group-based exercise; however, the difference between these subgroups was not statistically significant $\left(\chi^{2}=1.67\right.$, $P=0.43$ ).

Figure 3 shows the forest plot for fallers versus nonfallers. No overall heterogeneity was found between the studies $\left(I^{2}=0 \%\right)$. On completion of the intervention period, the exercise intervention reduced the risk of being a faller by $32 \%(\mathrm{RR}[95 \% \mathrm{CI}]=0.68$ [0.55-0.85]). Subgroup analysis was conducted, showing no difference between home-based and group-based exercise programs $\left(\chi^{2}=0.19\right.$, $P=0.91)$.
Comparison of two of the studies ${ }^{38,42}$ was also possible for the step test ${ }^{49}$ (Figure 4) and PPA ${ }^{50}$ (Figure 5). For the step test, no heterogeneity was found between the two studies $(R=0 \%)$. On completion of the intervention period, there was no significant difference in the results of the step test (number of steps) between the intervention and control groups (MD $[95 \% \mathrm{CI}]=0.51[-1.77$ to 2.78$]$ ). For the PPA, substantial heterogeneity was detected between the two studies that reported this outcome $\left(I^{2}=59 \%\right)$. However, the direction of effects of the two studies was not different. On completion of the intervention period, there was no significant difference in the results of the PPA between the intervention and control groups (MD $[95 \% \mathrm{CI}]=-0.10[-0.62$ to 0.42$])$.

\section{Discussion}

The purpose of this review was to evaluate the available evidence on the effectiveness of exercise or physical activity

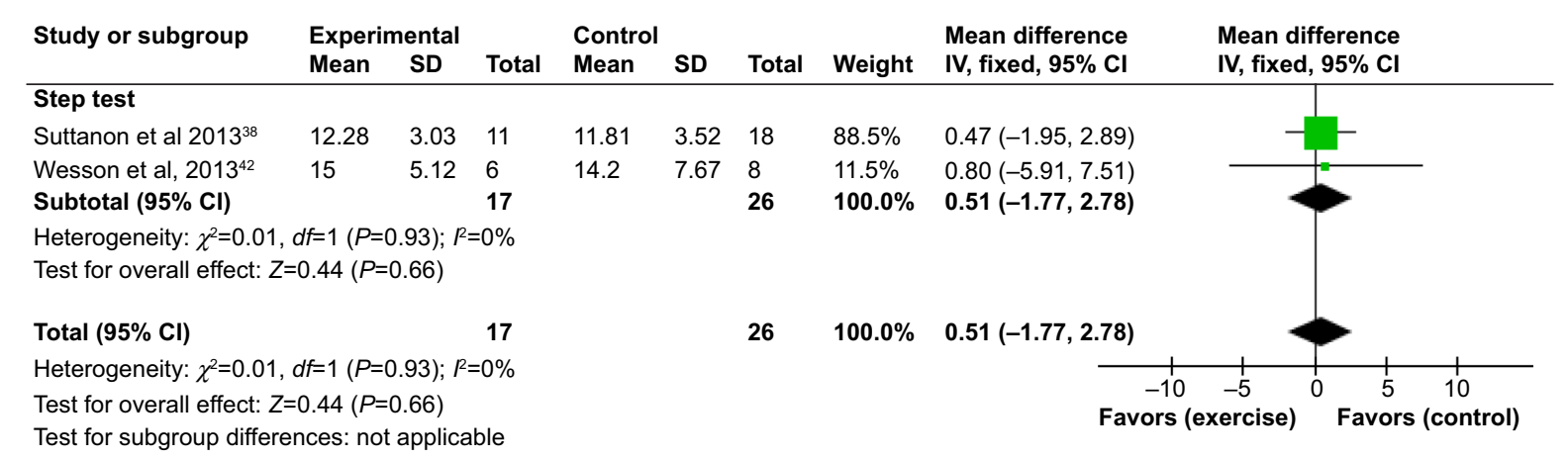

Figure 4 Forest plot of comparison: exercise versus usual care for step test. Abbreviations: SD, standard deviation; $\mathrm{Cl}$, confidence interval; IV, inverse variance. 


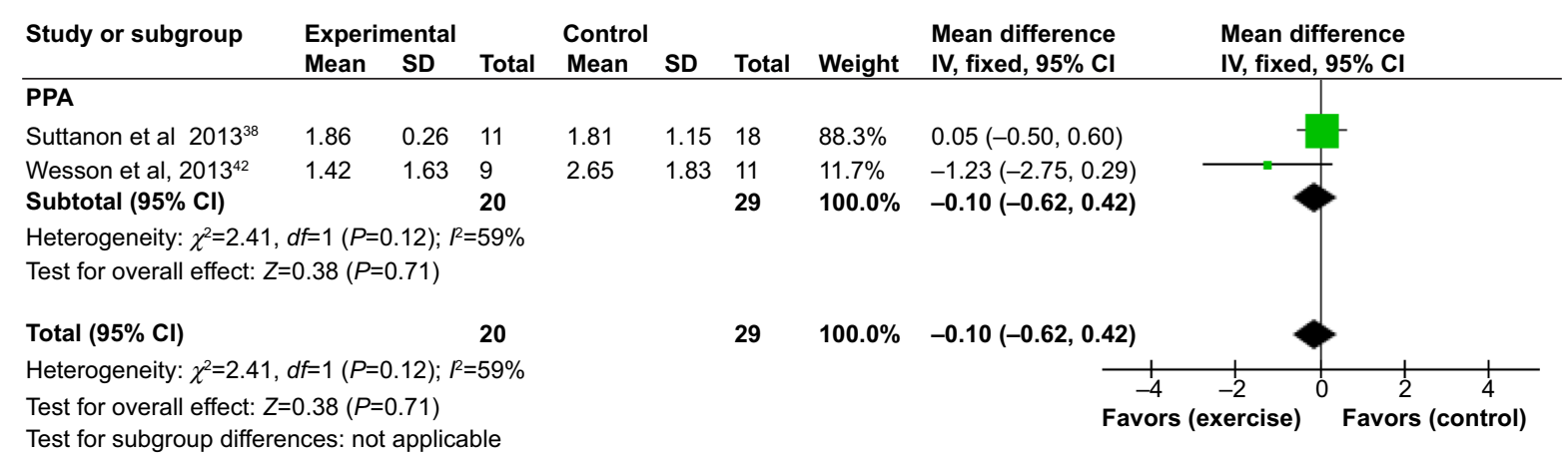

Figure 5 Forest plot of comparison: exercise versus usual care for PPA.

Abbreviations: PPA, physiological profile assessment; SD, standard deviation; $\mathrm{Cl}$, confidence interval; IV, inverse variance.

programs to reduce falls in older people with dementia who are living in the community. Data from three RCTs and one single pre- and post-test study with a total of 243 participants completing post testing were included. The meta-analyses demonstrated that exercise programs could potentially reduce falls for older people with dementia living in the community. Caution should be taken when interpreting the findings of our systematic review because of the inconsistencies between the studies, small sample sizes, as well as differences in outcome measures and the interventions used.

Overall, the exercise interventions achieved a significant reduction in the mean number of falls for home-based exercise and a $32 \%$ reduction in the risk of being a faller in the intervention group. The three RCTs included in the review were of high quality, although some of the studies were underpowered and follow-up limited, which has also been reported as a problem in falls prevention studies for older people without cognitive impairment living in the community. ${ }^{4,51}$ Limited comparison or effect was found for the other outcome measures used across the four studies. However, exercise programs that incorporate strength, balance and endurance, and progress in intensity over time have been shown to reduce falls in older people without cognitive impairment living in the community. ${ }^{15}$ This review and the small number of studies that have investigated this issue for older people living with dementia are a beginning to building on this evidence base. It appears that similar exercise interventions are likely to be beneficial in the reduction of falls and number of people falling, for older people living in the community with dementia. However, there may need to be some modifications, such as those utilized by Suttanon et $\mathrm{al}^{38}$ and Wesson et $\mathrm{a}^{142}$ including engagement of carers, regular contact by physiotherapist, more detailed exercise history in order to tailor the exercises to participant preferences, and a greater choice of exercises, which are challenging enough but can be implemented safely to accommodate the different cognitive and social circumstances.

Adherence to each exercise program ranged greatly between the studies, and two of the RCTs ${ }^{38,42}$ provided explicit reasons why exercise adherence was lower for some participants. They included lack of exercise previously, ill health, holidays, and the required support needed to exercise. Suttanon et $a^{52}$ conducted a detailed qualitative analysis, interviewing the participants and caregivers as an extension of the Suttanon et al's $\mathrm{s}^{38}$ included RCT, and found that preexisting or acute health conditions, a dislike of structured exercise, and caregiver causes (health and unavailable) were additional reasons for lower levels of adherence in the study. These reasons are similar to those of other studies that explored adherence to exercise for people with Alzheimer's disease and their caregivers. ${ }^{53,54}$ Teri et al ${ }^{54}$ found health issues such as dizziness and muscle strain while exercising, health problems for their caregivers, memory loss, confusion, and agitated or paranoid behavior were reasons for non-adherence in their study. When including people with dementia in future studies, there is likely benefit in addressing some of these factors in the implementation.

Another important finding with implications for future research in this area is the wide variety of secondary measures used in the included studies, limiting the potential for meaningful pooling of data and meta-analysis on these measures. An international working group for falls prevention studies has recommended in 2005 the need for improved definitions and standardization of key measures between fall prevention studies. ${ }^{43}$ These included a standard definition of a fall; daily recording of falls by participants, and a monthly follow-up phone call or face-to-face visit to resolve missing data and gain further falls and injury information; falls data should include number of falls, number of fallers/ non-fallers/frequent fallers, fall rate per person year, and time to first fall; data on injuries should be collected alongside falls 
data, including peripheral fracture rate per person year of follow-up, number of peripheral fractures, number of people sustaining fractures, and number of people sustaining multiple events. ${ }^{51}$ Other outcome measures suggested included the modified Falls Efficacy Scale (mFES) ${ }^{55}$ to measure the psychological consequences of falling, the Short Form 12 (SF12) version 2, and the European Quality of Life Instrument (EuroQoL EQ-5D) for Health Related Quality of Life. ${ }^{51}$ The ProFaNE team also recommended a 12-month follow-up.

In the four studies included in this systematic review, few of these recommendations were met. Only one study completed a 12-month follow-up; ${ }^{41}$ no study included the mFES, EQ-5D, or SF12-v2; and only two studies included a definition of a fall. ${ }^{38,42}$ Recording of falls and monthly follow-up were undertaken by all four studies and, as mentioned previously, falls were also measured in different ways across each study. These differences and the studies choosing different outcome measures to those recommended by ProFaNE may have been due to the target population being people with dementia and therefore the four studies used tools that were tested to be valid and reliable specifically for people with dementia. However, further research is needed to confirm this and additional recommendations for people with dementia living in the community who fall.

Two of the studies in this review used a diagnosis-related sample (Alzheimer's disease), while the other two studies included participants with cognitive impairment of any type. Given the differing etiology, pathology, signs and symptoms, and progression of disease between the various types of dementia, ${ }^{56}$ and also the differing rates of falls, ${ }^{5}$ there is value in future studies focusing on a specific diagnosis. However, given the small number of studies meeting the review criteria, we have included these studies with different sampling together in our meta-analyses.

\section{Limitations}

The strength of this review is that a systematic methodology was adopted to identify relevant studies and appraise the methodological quality and effectiveness of the interventions included and that meta-analyses were performed. However, there are several limitations to this review. Firstly, although six major databases were searched and strategies were used to supplement these, there is always a possibility that relevant studies were not included. The electronic search was limited to January 2000 to February 2014, and it is possible that studies published prior to this interval may be missing. However, Hauer et $\mathrm{al}^{35}$ had similar inclusion criteria with no limit to setting (ie, community living), and their search went back to 1966 . No further studies were found to meet our criteria, and therefore we are confident that the chances of this are slight. Because the search only included peer-reviewed literature, there is also the possibility that publication bias may have occurred because of unpublished, grey literature, or organizational (falls prevention) reports not being included. Language bias may have resulted because articles published in languages other than English were not included in the review; as fall prevention appears to be an issue facing many older people throughout the world, relevant articles written in languages other than English may have been excluded. The lack of studies and common outcome measures across the studies limit the ability to perform further meta-analyses, and the sub-grouped meta-analyses are preliminary and there is a need for further adequately powered trials to strengthen interpretation of the results of this review. The small sample sizes and shorter follow-up periods for some studies might also have been a limitation. The quality of the three RCTs, however, was high, which is a strength of the review, although caution must be taken when drawing conclusions because of the limitations discussed earlier.

\section{Conclusion}

This systematic review is the first of its kind to conduct meta-analyses to investigate the effectiveness of exercise interventions in reducing falls for older people with dementia living in the community. There is promising evidence that suggests falls prevention interventions, which include strength, balance, and endurance/mobility training and progress in intensity over time can assist in the reduction of falls in this population, although some caution is advised in interpreting these findings. The program can be undertaken either at home, in a group at a community center, or in a gymnasium. Further research is required, particularly RCTs using common outcome measures, standard follow-up periods of a minimum of 12 months, and appropriately powered sample sizes in order to determine the most appropriate falls reduction interventions for community-dwelling older people with dementia.

\section{Disclosure}

The authors report no conflicts of interest in this work.

\section{References}

1. Alzheimer's Disease International. Dementia Statistics; 2014. Available from: http://www.alz.co.uk/research/statistics. Accessed March 27, 2014.

2. Australian Institute of Health and Welfare. Australia's Health 2012. Australia's Health Series No. 13. Cat. No. AUS 156. Canberra: AIHW; 2012. 
3. Suttanon P, Hill K, Said C, Dodd K. Can balance exercise programmes improve balance and related physical performance measures in people with dementia? A systematic review. Eur Rev Aging Phys Act. 2010;7: $13-25$.

4. Gillespie L, Robertson M, Gillespie W, et al. Interventions for preventing falls in older people living in the community. Cochrane Database Syst Rev. 2012;9:CD007146

5. Allan L, Ballard C, Rowa E, Kenny R. Incidence and prediction of falls in dementia: a prospective study in older people. PLoS One. 2009; 4(5):e5521.

6. Eriksson S, Gustafson Y, Lundin-Olsson L. Risk factors for falls in people with and without a diagnose of dementia living in residential care facilities: a prospective study. Arch Gerontol Geriatr. 2008;46: 293-306.

7. Shaw F. Prevention of falls in older people with dementia. $J$ Neural Transm. 2007;114:1259-1264.

8. Moschny A, Platen P, Klaaßen-Mielke R, Trampisch U, Hinrichs T. Barriers to physical activity in older adults in Germany: a cross-sectional study. Int J Behav Nutr Phys Act. 2011;8:121.

9. Rasinaho M, Hirvensalo M, Leinonen R, Lintunen T, Rantanen T. Motives for and barriers to physical activity among older adults with mobility limitations. J Aging Phys Act. 2006;15:90-102.

10. Rowe M, Fehrenbach N. Injuries sustained by community-dwelling individuals with dementia. Clin Nurs Res. 2004;13(2):98-110.

11. Lord S. Predictors of nursing home placement and mortality of residents in intermediate care. Age Ageing. 1994;23(6):499-504.

12. Deandrea S, Lucenteforte E, Bravi F, Foschi R, La Vecchia C, Negri E. Risk factors for falls in community-dwelling older people: a systematic review and meta-analysis. Epidemiology. 2010;21(5):658-668.

13. Suttanon P, Hill K, Said C, Dodd K. A longitudinal study of change in falls risk and balance and mobility in healthy older people and people with Alzheimer Disease. Am J Phys Med Rehabil. 2013;92(8):676-685.

14. Guo J, Tsai Y, Liao J, Tu H, Huang C. Interventions to reduce the number of falls among older adults with/without cognitive impairment: an exploratory meta-analysis. Int J Geriatr Psychiatry. 2014;29(7):661-669.

15. Sherrington C, Tiedemann A, Fairhall N, Close J, Lord S. Exercise to prevent falls in older adults: an updated meta-analysis and best practice recommendations. N S W Public Health Bull. 2011;22(3-4):78-82.

16. Close J, Ellis M, Hooper R, Glucksman E, Jackson S, Swift C. Prevention of falls in the elderly trial (PROFET): a randomised controlled trial. Lancet. 1999;353:93-97.

17. Shaw F. Falls in older people with dementia. Geriatr Aging. 2003;6: 37-40.

18. Bunn F, Dickinson A, Simpson C, et al. Preventing falls among older people with mental health problems: a systematic review. BMC Nurs. 2014;13(4):1-15.

19. Härlein J, Dassen T, Halfens R, Heinze C. Fall risk factors in older people with dementia or cognitive impairment: a systematic review $J$ Adv Nurs. 2009;65(5):922-933.

20. Jensen L, Padilla R. Effectiveness of interventions to prevent falls in people with Alzheimer's disease and related dementias. Am J Occup Ther. 2011;65(5):532-540.

21. Kim D, Brown R, Ding E, Kiel D, Berry S. Dementia medications and risk of falls, syncope, and related adverse events meta-analysis of randomized controlled trials. J Am Geriatr Soc. 2011;59(6):1019-1031.

22. Meyer C, Hill S, Dow B, Synnot A, Hill K. Translating falls prevention knowledge to community-dwelling older PLWD: a mixed-method systematic review. Gerontologist. 2013. doi: 10.1093/geron/gnt127.

23. Muir S, Gopaul K, Odasso M. The role of cognitive impairment in fall risk among older adults: a systematic review and meta-analysis. Age Ageing. 2012;41:299-308.

24. Oliver D, Connelly JB, Victor CR, et al. Strategies to prevent falls and fractures in hospitals and care homes and effect of cognitive impairment: systematic review and meta-analyses. BMJ. 2007;334(7584):82.

25. Winter $\mathrm{H}$, Watt K, Peel N. Falls prevention interventions for communitydwelling older persons with cognitive impairment: a systematic review. Int Psychogeriatr. 2013;25(2):215-227.

26. Perkins C. Dementia and falling. N Z Fam Physician. 2008;35(1):44-46.
27. Taylor M, Delbaere K, Close J, Lord S. Managing falls in older patients with cognitive impairment. Aging Health. 2012;8(6):573-588.

28. Bowes A, Dawson A, Jepson R, McCabe L. Physical activity for people with dementia: a scoping study. BMC Geriatr. 2013;13:129.

29. Christofoletti G, Oliani M, Gobbi S, Stella F. Effects of motor intervention in elderly patients with dementia: an analysis of randomized controlled trials. Top Geriatr Rehabil. 2007;23(2):149-154.

30. Forbes D, Thiessen E, Blake C, Forbes S. Exercise programs for people with dementia. Cochrane Database Syst Rev. 2013;12: CD006489.

31. Heyn P, Abreu B, Ottenbacher K. The effects of exercise training on elderly persons with cognitive impairment and dementia: a metaanalysis. Arch Phys Med Rehabil. 2004;85:1694-1704.

32. Kearney F, Harwood R, Gladman J, Lincoln N, Masud T. The relationship between executive function and falls and gait abnormalities in older adults: a systematic review. Dement Geriatr Cogn Disord. 2013; $36(1): 20-35$.

33. Littbrand H, Stenvall M, Rosendahl E. Applicability and effects of physical exercise on physical and cognitive functions and activities of daily living among people with dementia: a systematic review. Am J Phys Med Rehabil. 2011;90:495-518.

34. Pitkälä K, Savikko N, Poysti M, Strandberg T, Laakkonen M. Efficacy of physical exercise intervention on mobility and physical functioning in older people with dementia: a systematic review. Exp Gerontol. 2013;48: 85-93.

35. Hauer K, Becker C, Lindemann U, Beyer N. Effectiveness of physical training on motor performance and fall prevention in cognitively impaired older persons: a systematic review. Am J Phys Med Rehabil. 2006; $85: 847-857$

36. Liberati A, Altman D, Tetzlaff J, et al. The PRISMA statement for reporting systematic reviews and meta-analyses of studies that evaluate health care interventions: explanation and elaboration. BMJ. 2009; 339:b2700.

37. Higgins J, Altman D, Gøtzsche P, et al. The Cochrane Collaboration's tool for assessing risk of bias in randomised trials. $B M J .2011$; 343:d5928.

38. Suttanon P, Hill KD, Said CM, et al. Feasibility, safety and preliminary evidence of the effectiveness of a home-based exercise programme for older people with Alzheimer's disease: a pilot randomized controlled trial. Clin Rehabil. 2013;27(5):427-438.

39. DerSimonian R, Laird N. Meta-analysis in clinical trials. Control Clin Trials. 1986;7(3):177-188.

40. Higgins J, Green S. Cochrane Handbook for Systematic Reviews of Interventions Version 5.1.0 [updated March 2011]. The Cochrane Collaboration; 2011. Available from: www.cochrane-handbook.org.

41. Pitkälä KH, Pöysti MM, Laakkonen ML, et al. Effects of the Finnish Alzheimer's disease exercise trial (FINALEX). JAMA Intern Med. 2013; 173(10):894-901.

42. Wesson J, Clemson L, Brodaty H, et al. A feasibility study and pilot randomised trial of a tailored prevention program to reduce falls in older people with mild dementia. BMC Geriatr. 2013;13:89.

43. Mackintosh S, Sheppard L. A pilot falls-prevention programme for older people with dementia from a predominantly Italian background. Hong Kong Phys J. 2005;23:20-26.

44. Robertson M, Campbell AJ, Gardner M, Devlin N. Preventing injuries in older people by preventing falls: a meta-analysis of individual-level data. J Am Geriatr Soc. 2002;50(5):905-911.

45. World Health Organisation. Global Recommendations on Physical Activity for Health 65 Years and Above; 2011. Available from: http:// www.who.int/dietphysicalactivity/physical-activity-recommendations65years.pdf. Accessed February 18, 2012.

46. Sims J, Hill K, Hunt S, Haralambous B. Physical activity recommendations for older Australians. Australas J Ageing. 2010;29(2): $81-87$.

47. Suttanon P. Balance and Falls Risk in Alzheimer's Disease: Magnitude of the Problem and Feasibility and Effects of a Balance Exercise Program. Bundoora, VIC: School of Physiotherapy, Faculty of Health Sciences, La Trobe University; 2011. 
48. Vogler C, Sherrington C, Ogle S, Lord S. Reducing risk of falling in older people discharged from hospital: a randomized controlled trial comparing seated exercises, weight-bearing exercises, and social visits. Arch Phys Med Rehabil. 2009;90:1317-1324.

49. Hill K, Bernhardt J, McGann A, Maltese D, Berkovits D. A new test of dynamic standing balance for stroke patients: reliability, validity, and comparison with healthy elderly. Physiother Canada. 1996;48: 257-262.

50. Lord S, Menz H, Tiedemann A. A physiological profile approach to falls risk assessment and prevention. Phys Ther. 2003;83(3):237-252.

51. Lamb S, Jørstad-Stein E, Hauer K, Becker C, on behalf of the Prevention of Falls Network Europe and Outcome Consensus Group. Development of a common outcome data set for fall injury prevention trials: the Prevention of Falls Network Europe Consensus. J Am Geriatr Soc. 2005;53(9):1618-1622.
52. Suttanon P, Hill K, Said C, Byrne K, Dodd K. Factors influencing commencement and adherence to a home-based balance exercise program for reducing risk of falls: perceptions of people with Alzheimer's disease and their caregivers. Int Psychogeriatr. 2012;24(7): $1172-1182$.

53. Teri L, Gibbons LE, McCurry SM, et al. Exercise plus behavioral management in patients with Alzheimer disease: a randomized controlled trial. JAMA. 2003;290(15):2015-2022.

54. Teri L, McCurry S, Buchner D, et al. Exercise and activity level in Alzheimer's disease: a potential treatment focus. J Rehabil Res Dev. 1998;35(4):411-419.

55. Hill K, Schwarz J, Kalogeropoulos A, Gibson S. Fear of falling revisited. Arch Phys Med Rehabil. 1996;77(10):1025-1029.

56. Bhidayasiri R. Atypical dementia: when it is not Alzheimer's disease. J Med Assoc Thai. 2007;90(10):2222-2232.
Clinical Interventions in Aging

\section{Publish your work in this journal}

Clinical Interventions in Aging is an international, peer-reviewed journal focusing on evidence-based reports on the value or lack thereof of treatments intended to prevent or delay the onset of maladaptive correlates of aging in human beings. This journal is indexed on PubMed Central, MedLine,

\section{Dovepress}

CAS, Scopus and the Elsevier Bibliographic databases. The manuscript management system is completely online and includes a very quick and fair peer-review system, which is all easy to use. Visit http://www.dovepress. com/testimonials.php to read real quotes from published authors. 